\title{
Multiple Positive Solutions of a Singular Emden-Fowler Type Problem for Second-Order Impulsive Differential Systems
}

\author{
Eun Kyoung Lee and Yong-Hoon Lee \\ Department of Mathematics, Pusan National University, Busan 609-735, Republic of Korea \\ Correspondence should be addressed to Yong-Hoon Lee, yhlee@pusan.ac.kr \\ Received 14 May 2010; Accepted 26 July 2010 \\ Academic Editor: Feliz Manuel Minhós
}

Copyright (C) 2011 E. K. Lee and Y.-H. Lee. This is an open access article distributed under the Creative Commons Attribution License, which permits unrestricted use, distribution, and reproduction in any medium, provided the original work is properly cited.

This paper studies the existence, and multiplicity of positive solutions of a singular boundary value problem for second-order differential systems with impulse effects. By using the upper and lower solutions method and fixed point index arguments, criteria of the multiplicity, existence and nonexistence of positive solutions with respect to parameters given in the system are established.

\section{Introduction}

In this paper, we consider systems of impulsive differential equations of the form

$$
\begin{gathered}
u^{\prime \prime}(t)+\lambda h_{1}(t) f(u(t), v(t))=0, \quad t \in(0,1), \quad t \neq t_{1}, \\
v^{\prime \prime}(t)+\mu h_{2}(t) g(u(t), v(t))=0, \quad t \in(0,1), \quad t \neq t_{1}, \\
\left.\Delta u\right|_{t=t_{1}}=I_{u}\left(u\left(t_{1}\right)\right),\left.\quad \Delta v\right|_{t=t_{1}}=I_{v}\left(v\left(t_{1}\right)\right), \\
\left.\Delta u^{\prime}\right|_{t=t_{1}}=N_{u}\left(u\left(t_{1}\right)\right),\left.\quad \Delta v^{\prime}\right|_{t=t_{1}}=N_{v}\left(v\left(t_{1}\right)\right), \\
u(0)=a \geq 0, \quad v(0)=b \geq 0, \quad u(1)=c \geq 0, \quad v(1)=d \geq 0,
\end{gathered}
$$

where $\lambda, \mu$ are positive real parameters, $\left.\Delta u\right|_{t=t_{1}}=u\left(t_{1}^{+}\right)-u\left(t_{1}\right)$, and $\left.\Delta u^{\prime}\right|_{t=t_{1}}=u^{\prime}\left(t_{1}^{+}\right)-u^{\prime}\left(t_{1}^{-}\right)$. Throughout this paper, we assume $f, g \in \mathrm{C}\left(\mathbb{R}_{+}^{2}, \mathbb{R}_{+}\right)$with $f(0,0)=0=g(0,0)$ and $f(u, v)>$ $0, g(u, v)>0$ for all $(u, v) \neq(0,0), I_{u}, I_{v} \in C\left(\mathbb{R}_{+}, \mathbb{R}\right)$ satisfying $I_{u}(0)=0=I_{v}(0), N_{u}, N_{v} \in$ $C\left(\mathbb{R}_{+},(-\infty, 0]\right)$, and $h_{i} \in C((0,1),(0, \infty)), i=1,2$. Here we denote $\mathbb{R}_{+}=[0, \infty)$. We note that 
$h_{i}$ may be singular at $t=0$ and/or 1 . Let $J=[0,1], J^{\prime}=[0,1] \backslash\left\{0,1, t_{1}\right\}, P C[0,1]=\{u \mid$ $u:[0,1] \rightarrow \mathbb{R}$ be continuous at $t \neq t_{1}$, left continuous at $t=t_{1}$, and its right-hand limit at $t=t_{1}$ exists $\}$ and $X=P C[0,1] \times P C[0,1]$. Then $P C[0,1]$ and $X$ are Banach spaces with norm $\|u\|=\sup _{t \in[0,1]}|u(t)|$ and $\|(u, v)\|=\|u\|+\|v\|$, respectively. The solution of problem $(P)$ means $(u, v) \in X \cap\left(C^{2}\left(J^{\prime}\right) \times C^{2}\left(J^{\prime}\right)\right)$ which satisfies $(P)$.

Recently, several works have been devoted to the study of second-order impulsive differential systems. See, for example [1-6], and references therein. In Particular, E.K. Lee and Y.H. Lee [3] studied problem $(P)$ when $f$ and $g$ satisfy $f(0,0)>0$ and $g(0,0)>0$. More precisely, let us consider the following assumptions.

$\left(D_{1}\right) \int_{0}^{1} s(1-s) h_{i}(s) d s<\infty$, for $i=1,2$.

$\left(D_{2}\right) t_{1} N_{u}(u) \leq I_{u}(u) \leq-\left(1-t_{1}\right) N_{u}(u)$ and $t_{1} N_{v}(v) \leq I_{v}(v) \leq-\left(1-t_{1}\right) N_{v}(v)$.

$\left(D_{3}\right) u+I_{u}(u)$ and $v+I_{v}(v)$ are nondecreasing.

$\left(D_{4}\right) N_{u, \infty}=\lim _{u \rightarrow \infty}\left|N_{u}(u)\right| / u<1$ and $N_{v, \infty}=\lim _{v \rightarrow \infty}\left|N_{v}(v)\right| / v<1$.

$\left(D_{5}\right) f_{\infty}=\lim _{u+v \rightarrow \infty} f(u, v) / u+v=\infty$ and $g_{\infty}=\lim _{u+v \rightarrow \infty} g(u, v) / u+v=\infty$.

$\left(D_{6}\right) f$ and $g$ are nondecreasing on $\mathbb{R}_{+}^{2}$, that is, $f\left(u_{1}, v_{1}\right) \leq f\left(u_{2}, v_{2}\right)$ and $g\left(u_{1}, v_{1}\right) \leq$ $g\left(u_{2}, v_{2}\right)$ whenever $\left(u_{1}, v_{1}\right) \leq\left(u_{2}, v_{2}\right)$, where inequality on $\mathbb{R}_{+}^{2}$ can be understood componentwise.

Under the above assumptions, they proved that there exists a continuous curve $\Gamma$ splitting $\mathbb{R}_{+}^{2} \backslash\{(0,0)\}$ into two disjoint subsets $\mathcal{O}_{1}$ and $\mathcal{O}_{2}$ such that problem (3.20) has at least two positive solutions for $(\lambda, \mu) \in \mathcal{O}_{1}$, at least one positive solution for $(\lambda, \mu) \in \Gamma$, and no solution for $(\lambda, \mu) \in \mathcal{O}_{2}$.

The aim of this paper is to study generalized Emden-Fowler-type problem for $(P)$, that is, $f$ and $g$ satisfy $f(0,0)=0$ and $g(0,0)=0$, respectively. In this case, we obtain two interesting results. First, for Dirichlet boundary condition, that is, $a=b=c=d=0$, assuming $\left(D_{1}\right),\left(D_{2}\right)$ and

$$
\begin{aligned}
& \left(D_{4}^{\prime}\right) N_{u, 0}=\lim _{u \rightarrow 0}\left|N_{u}(u)\right| / u<1 / 2 \text { and } N_{v, 0}=\lim _{v \rightarrow 0}\left|N_{v}(v)\right| / v<1 / 2, \\
& \left(D_{5}^{\prime}\right) f_{\infty}=\infty, g_{\infty}=\infty \text { and } f_{0}=\lim _{u+v \rightarrow 0} f(u, v) / u+v=0, g_{0}=\lim _{u+v \rightarrow 0} g(u, v) / u+v=0,
\end{aligned}
$$

we prove that problem $(P)$ has at least one positive solution for all $(\lambda, \mu) \in \mathbb{R}_{+}^{2} \backslash\{(0,0)\}$. On the other hand, for two-point boundary condition, that is, $c>a$ and $d>b$, assuming $\left(D_{1}\right) \sim\left(D_{6}\right)$, we prove that there exists a continuous curve $\Gamma_{0}$ splitting $\mathbb{R}_{+}^{2} \backslash\{(0,0)\}$ into two disjoint subsets $\mathcal{O}_{0,1}$ and $\mathcal{O}_{0,2}$ and there exists a subset $\mathcal{O} \subset \mathcal{O}_{0,1}$ such that problem $(P)$ has at least two positive solutions for $(\lambda, \mu) \in \mathcal{O}$, at least one positive solution for $(\lambda, \mu) \in\left(\mathcal{O}_{0,1} \backslash \mathcal{O}\right) \cup \Gamma_{0}$, and no solution for $(\lambda, \mu) \in \mathcal{O}_{0,2}$.

Our technique of proofs is mainly employed by the upper and lower solutions method and several fixed point index theorems.

The paper is organized as follows: in Section 2, we introduce and prove two types of upper and lower solutions and related theorems, one for singular systems with no impulse effect and the other for singular impulsive systems and then introduce several fixed point index theorems for later use. In Section 3, we prove an existence result for Dirichlet boundary value problems and existence and nonexistence part of the result for two-point boundary value problems. In Section 4, we prove the existence of the second positive solution for two point boundary value problems. Finally, in Section 5, we apply main results to prove some theorems of existence, nonexistence, and multiplicity of positive radial solutions for impulsive semilinear elliptic problems. 


\section{Preliminary}

In this section, we introduce two types of fundamental theorems of upper and lower solutions method for a singular system with no impulse effect and an impulsive system and then introduce several well-known fixed point index theorems. We first give definition $\mathrm{s}$ of somewhat general type of upper and lower solutions for the following singular system:

$$
\begin{gathered}
u^{\prime \prime}(t)+F(t, u(t), v(t))=0, \quad t \in(0,1), \\
v^{\prime \prime}(t)+G(t, u(t), v(t))=0, \quad t \in(0,1), \\
u(0)=A, \quad u(1)=C, \quad v(0)=B, \quad v(1)=D,
\end{gathered}
$$

where $F, G:(0,1) \times \mathbb{R} \times \mathbb{R} \rightarrow \mathbb{R}$ are continuous.

Definition 2.1. We say that $\left(\alpha_{u}, \alpha_{v}\right) \in C[0,1] \times C[0,1]$ is a G-lower solution of $(H)$ if $\left(\alpha_{u}, \alpha_{v}\right) \in$ $C^{2}(0,1) \times C^{2}(0,1)$ except at finite points $\tau_{1}, \ldots, \tau_{n}$ with $0<\tau_{1}<\ldots<\tau_{n}<1$ such that

$\left(L_{1}\right)$ at each $\tau_{i}$, there exist $\left(\alpha_{u}^{\prime}\left(\tau_{i}^{-}\right), \alpha_{v}^{\prime}\left(\tau_{i}^{-}\right)\right),\left(\alpha_{u}^{\prime}\left(\tau_{i}^{+}\right), \alpha_{v}^{\prime}\left(\tau_{i}^{+}\right)\right)$such that $\alpha_{u}^{\prime}\left(\tau_{i}^{-}\right) \leq$ $\alpha_{u}^{\prime}\left(\tau_{i}^{+}\right), \alpha_{v}^{\prime}\left(\tau_{i}^{-}\right) \leq \alpha_{v}^{\prime}\left(\tau_{i}^{+}\right)$, and

$\left(L_{2}\right)$

$$
\begin{gathered}
\alpha_{u}^{\prime \prime}(t)+F\left(t, \alpha_{u}(t), \alpha_{v}(t)\right) \geq 0, \\
\alpha_{v}^{\prime \prime}(t)+G\left(t, \alpha_{u}(t), \alpha_{v}(t)\right) \geq 0, \quad t \in \frac{(0,1)}{\left\{\tau_{1}, \ldots, \tau_{n}\right\}}, \\
\alpha_{u}(0) \leq A, \quad \alpha_{u}(1) \leq C, \\
\alpha_{v}(0) \leq B, \quad \alpha_{v}(1) \leq D .
\end{gathered}
$$

We also say that $\left(\beta_{u}, \beta_{v}\right) \in C[0,1] \times C[0,1]$ is a G-upper solution of the problem $(H)$ if $\left(\beta_{u}, \beta_{v}\right) \in C^{2}(0,1) \times C^{2}(0,1)$ except at finite points $\sigma_{1}, \ldots, \sigma_{m}$ with $0<\sigma_{1}<\cdots<\sigma_{m}<1$ such that

$\left(U_{1}\right)$ at each $\sigma_{i}$, there exist $\left(\beta_{u}^{\prime}\left(\sigma_{j}^{-}\right), \beta_{v}^{\prime}\left(\sigma_{j}^{-}\right)\right),\left(\beta_{u}^{\prime}\left(\sigma_{j}^{+}\right), \beta_{v}^{\prime}\left(\sigma_{j}^{+}\right)\right)$such that $\beta_{u}^{\prime}\left(\sigma_{j}^{-}\right) \geq$ $\beta_{u}^{\prime}\left(\sigma_{j}^{+}\right), \beta_{v}^{\prime}\left(\sigma_{j}^{-}\right) \geq \beta_{v}^{\prime}\left(\sigma_{j}^{+}\right)$, and

$\left(U_{2}\right)$

$$
\begin{gathered}
\beta_{u}^{\prime \prime}(t)+F\left(t, \beta_{u}(t), \beta_{v}(t)\right) \leq 0, \\
\beta_{v}^{\prime \prime}(t)+G\left(t, \beta_{u}(t), \beta_{v}(t)\right) \leq 0, \quad t \in \frac{(0,1)}{\left\{\sigma_{1}, \ldots, \sigma_{n}\right\}}, \\
\beta_{u}(0) \geq A, \quad \beta_{u}(1) \geq C, \\
\beta_{v}(0) \geq B, \quad \beta_{v}(1) \geq D .
\end{gathered}
$$

For the proof of the fundamental theorem on G-upper and G-lower solutions for problem $(H)$, we need the following lemma. One may refer to [7] for the proof. 
Lemma 2.2. Let $F, G: D \rightarrow \mathbb{R}$ be continuous functions and $D \subset(0,1) \times \mathbb{R} \times \mathbb{R}$. Assume that there exist $h_{F}, h_{G} \in C\left((0,1), \mathbb{R}^{+}\right)$such that

$$
|F(t, u, v)| \leq h_{F}(t), \quad|G(t, u, v)| \leq h_{G}(t),
$$

for all $(t, u, v) \in(0,1) \times \mathbb{R} \times \mathbb{R}$, and

$$
\int_{0}^{1} s(1-s) h_{F}(s) d s+\int_{0}^{1} s(1-s) h_{G}(s) d s<\infty
$$

Then problem $(H)$ has a solution.

Let $D_{\alpha}^{\beta}=\left\{(t, u, v) \mid\left(\alpha_{u}(t), \alpha_{v}(t)\right) \leq(u, v) \leq\left(\beta_{u}(t), \beta_{v}(t)\right), t \in[0,1]\right\}$. Then the fundamental theorem of G-upper and G-lower solutions for singular problem $(H)$ is given as follows.

Theorem 2.3. Let $\left(\alpha_{u}, \alpha_{v}\right)$ and $\left(\beta_{u}, \beta_{v}\right)$ be a G-lower solution and a G-upper solution of problem $(H)$, respectively, such that

$$
\left(a_{1}\right)\left(\alpha_{u}(t), \alpha_{v}(t)\right) \leq\left(\beta_{u}(t), \beta_{v}(t)\right) \text { for all } t \in[0,1] .
$$

Assume also that there exist $h_{F}, h_{G} \in C\left((0,1), \mathbb{R}^{+}\right)$such that

$$
\begin{aligned}
& \left(a_{2}\right)|F(t, u, v)| \leq h_{F}(t) \text { and }|G(t, u, v)| \leq h_{G}(t) \text { for all }(t, u, v) \in D_{\alpha}^{\beta} ; \\
& \left(a_{3}\right) \int_{0}^{1} s(1-s) h_{F}(s) d s+\int_{0}^{1} s(1-s) h_{G}(s) d s<\infty ; \\
& \left(a_{4}\right) F\left(t, u, v_{1}\right) \leq F\left(t, u, v_{2}\right) \text {, whenever } v_{1} \leq v_{2} \text { and } G\left(t, u_{1}, v\right) \leq G\left(t, u_{2}, v\right) \text {, whenever } u_{1} \leq \\
& \quad u_{2} .
\end{aligned}
$$

Then problem $(H)$ has at least one solution $(u, v)$ such that

$$
\left(\alpha_{u}(t), \alpha_{v}(t)\right) \leq(u(t), v(t)) \leq\left(\beta_{u}(t), \beta_{v}(t)\right), \quad \forall t \in[0,1]
$$

Proof. Define a modified function of $F$ as follows:

$$
F_{*}(t, u, v)= \begin{cases}F\left(t, \beta_{u}(t), v\right)-\frac{u-\beta_{u}(t)}{1}+u^{2} & \text { if } u>\beta_{u}(t) \\ F(t, u, v) & \text { if } \alpha_{u}(t) \leq u \leq \beta_{u}(t) \\ F\left(t, \alpha_{u}(t), v\right)-\frac{u-\alpha_{u}(t)}{1}+u^{2} & \text { if } u<\alpha_{u}(t)\end{cases}
$$




$$
\begin{gathered}
F^{*}(t, u, v)= \begin{cases}F_{*}\left(t, u, \beta_{v}(t)\right) & \text { if } v>\beta_{v}(t), \\
F_{*}(t, u, v) & \text { if } \alpha_{v}(t) \leq v \leq \beta_{v}(t), \\
F_{*}\left(t, u, \alpha_{v}(t)\right) & \text { if } v<\alpha_{v}(t),\end{cases} \\
G_{*}(t, u, v)= \begin{cases}G\left(t, \beta_{u}(t), v\right) & \text { if } u>\beta_{u}(t), \\
G(t, u, v) & \text { if } \alpha_{u}(t) \leq u \leq \beta_{u}(t), \\
G\left(t, \alpha_{u}(t), v\right) & \text { if } u<\alpha_{u}(t),\end{cases} \\
G^{*}(t, u, v)= \begin{cases}G_{*}\left(t, u, \beta_{v}(t),\right)-\frac{v-\beta_{v}(t)}{1}+v^{2} & \text { if } v>\beta_{v}(t), \\
G_{*}(t, u, v) & \text { if } \alpha_{v}(t) \leq v \leq \beta_{v}(t), \\
G_{*}\left(t, u, \alpha_{v}(t)\right)-\frac{v-\alpha_{v}(t)}{1}+v^{2} & \text { if } v<\alpha_{v}(t) .\end{cases}
\end{gathered}
$$

Then $F^{*}, G^{*}:(0,1) \times \mathbb{R} \times \mathbb{R} \rightarrow \mathbb{R}$ are continuous and

$$
\begin{aligned}
& \left|F^{*}(t, u, v)\right| \leq m\left(\alpha_{u}, \beta_{u}\right)+h_{F}(t), \\
& \left|G^{*}(t, u, v)\right| \leq m\left(\alpha_{v}, \beta_{v}\right)+h_{G}(t),
\end{aligned}
$$

for all $(t, u, v) \in(0,1) \times \mathbb{R} \times \mathbb{R}$, where $m(\alpha, \beta)=\|\alpha\|+\|\beta\|+1$. For the problem

$$
\begin{gathered}
u^{\prime \prime}(t)+F^{*}(t, u(t), v(t))=0, \\
v^{\prime \prime}(t)+G^{*}(t, u(t), v(t))=0, \quad t \in(0,1) \\
u(0)=A, \quad u(1)=C, \quad v(0)=B, \quad v(1)=D,
\end{gathered}
$$

Lemma 2.2 guarantees the existence of solutions of problem $(M)$ and thus it is enough to prove that any solution $(u, v)$ of problem $(M)$ satisfies

$$
\left(\alpha_{u}(t), \alpha_{v}(t)\right) \leq(u(t), v(t)) \leq\left(\beta_{u}(t), \beta_{v}(t)\right), \quad \forall t \in[0,1]
$$

Suppose, on the contrary, $\left(\alpha_{u}, \alpha_{v}\right) \leq(u, v)$, so we consider the case $\alpha_{u} \leq u$. Let $\left(\alpha_{u}-u\right)\left(t_{0}\right)=$ $\max _{t \in[0,1]}\left(\alpha_{u}-u\right)(t)>0$. If $t_{0} \in(0,1) \backslash\left\{\tau_{1}, \ldots, \tau_{n}\right\}$, then $\left(\alpha_{u}-u\right)^{\prime \prime}\left(t_{0}\right) \leq 0$. We consider two 
cases. First, if $\alpha_{v}\left(t_{0}\right) \leq v\left(t_{0}\right)$, then by $\alpha_{u}\left(t_{0}\right)>u\left(t_{0}\right)$ and condition $\left(a_{4}\right)$,

$$
\begin{aligned}
0 & \geq\left(\alpha_{u}-u\right)^{\prime \prime}\left(t_{0}\right)=\alpha_{u}^{\prime \prime}\left(t_{0}\right)+F^{*}\left(t_{0}, u\left(t_{0}\right), v\left(t_{0}\right)\right) \\
& =\alpha_{u}^{\prime \prime}\left(t_{0}\right)+F\left(t_{0}, \alpha_{u}\left(t_{0}\right), v\left(t_{0}\right)\right)-\frac{u\left(t_{0}\right)-\alpha_{u}\left(t_{0}\right)}{1+u^{2}\left(t_{0}\right)} \\
& \geq \alpha_{u}^{\prime \prime}\left(t_{0}\right)+F\left(t_{0}, \alpha_{u}\left(t_{0}\right), \alpha_{v}\left(t_{0}\right)\right)-\frac{u\left(t_{0}\right)-\alpha_{u}\left(t_{0}\right)}{1+u^{2}\left(t_{0}\right)} \\
& \geq \frac{\alpha_{u}\left(t_{0}\right)-u\left(t_{0}\right)}{1+u^{2}\left(t_{0}\right)}>0
\end{aligned}
$$

which is a contradiction. Next, if $\alpha_{v}\left(t_{0}\right)>v\left(t_{0}\right)$, then by the definition of $F^{*}$,

$$
\begin{aligned}
0 & \geq\left(\alpha_{u}-u\right)^{\prime \prime}\left(t_{0}\right)=\alpha_{u}^{\prime \prime}\left(t_{0}\right)+F^{*}\left(t_{0}, u\left(t_{0}\right), v\left(t_{0}\right)\right) \\
& =\alpha_{u}^{\prime \prime}\left(t_{0}\right)+F\left(t_{0}, \alpha_{u}\left(t_{0}\right), \alpha_{v}\left(t_{0}\right)\right)-\frac{u\left(t_{0}\right)-\alpha_{u}\left(t_{0}\right)}{1+u^{2}\left(t_{0}\right)}>0,
\end{aligned}
$$

which is also a contradiction. If $t_{0}=\tau_{i}$ for some $i=1, \ldots, n$, then since $\alpha_{u}-u$ attains its positive maximum at $\tau_{i}$,

$$
\left(\alpha_{u}-u\right)^{\prime}\left(\tau_{i}^{-}\right) \geq 0, \quad\left(\alpha_{u}-u\right)^{\prime}\left(\tau_{i}^{+}\right) \leq 0
$$

If $\left(\alpha_{u}-u\right)^{\prime}\left(\tau_{i}^{-}\right)>0$, then

$$
0<\left(\alpha_{u}-u\right)^{\prime}\left(\tau_{i}^{-}\right)-\left(\alpha_{u}-u\right)^{\prime}\left(\tau_{i}^{+}\right)=\alpha_{u}^{\prime}\left(\tau_{i}^{-}\right)-\alpha_{u^{\prime}}\left(\tau_{i}^{+}\right)
$$

This leads a contradiction to the definition of $G$-lower solution. If $\left(\alpha_{u}-u\right)^{\prime}\left(\tau_{i}^{-}\right)=0$, then there exists $\delta>0$ such that for all $t \in\left(\tau_{i}-\delta, \tau_{i}\right)$,

$$
\left(\alpha_{u}-u\right)(t)>0, \quad\left(\alpha_{u}-u\right)^{\prime}(t) \geq 0, \quad\left(\alpha_{u}-u\right)^{\prime \prime}(t) \leq 0 .
$$

For $t \in\left(\tau_{i}-\delta, \tau_{i}\right)$, if $\alpha_{v}(t) \leq v(t)$, then by $\alpha_{u}(t)>u(t)$ and condition $\left(a_{4}\right)$,

$$
\begin{aligned}
0 & \geq\left(\alpha_{u}-u\right)^{\prime \prime}(t)=\alpha_{u}^{\prime \prime}(t)+F^{*}(t, u(t), v(t)) \\
& =\alpha_{u}^{\prime \prime}(t)+F\left(t, \alpha_{u}(t), v(t)\right)-\frac{u(t)-\alpha_{u}(t)}{1+u^{2}(t)} \\
& \geq \alpha_{u}^{\prime \prime}(t)+F\left(t, \alpha_{u}(t), \alpha_{v}(t)\right)-\frac{u(t)-\alpha_{u}(t)}{1+u^{2}(t)} \\
& \geq \frac{\alpha_{u}(t)-u(t)}{1+u^{2}(t)}>0
\end{aligned}
$$


which is a contradiction. If $\alpha_{v}(t)>v(t)$, then by definition of $F^{*}$,

$$
\begin{aligned}
0 & \geq\left(\alpha_{u}-u\right)^{\prime \prime}(t)=\alpha_{u}^{\prime \prime}(t)+F^{*}(t, u(t), v(t)) \\
& =\alpha_{u}^{\prime \prime}(t)+F\left(t, \alpha_{u}(t), \alpha_{v}(t)\right)-\frac{u(t)-\alpha_{u}(t)}{1+u^{2}(t)}>0,
\end{aligned}
$$

which is a contradiction. If $t_{0}=0$ or 1 , then

$$
\begin{aligned}
& 0<\left(\alpha_{u}-u\right)(0)=\alpha_{u}(0)-A \leq 0, \\
& 0<\left(\alpha_{u}-u\right)(1)=\alpha_{u}(1)-C \leq 0,
\end{aligned}
$$

which is a contradiction. Similarly, we get contradictions for the case $\alpha_{v} \not \leq v$. The proof for $(u, v) \leq\left(\beta_{u}, \beta_{v}\right)$ can be done by similar fashion.

Now we introduce definition and fundamental theorem of upper and lower solutions for impulsive differential systems of the form

$$
\begin{gathered}
u^{\prime \prime}(t)+F(t, u(t), v(t))=0, \quad t \neq t_{1}, \quad t \in(0,1), \\
u^{\prime \prime}(t)+G(t, u(t), v(t))=0, \quad t \neq t_{1}, \quad t \in(0,1), \\
\left.\Delta u\right|_{t=t_{1}}=I_{u}\left(u\left(t_{1}\right)\right),\left.\quad \Delta v\right|_{t=t_{1}}=I_{v}\left(v\left(t_{1}\right)\right), \\
\left.\Delta u^{\prime}\right|_{t=t_{1}}=N_{u}\left(u\left(t_{1}\right)\right),\left.\quad \Delta v^{\prime}\right|_{t=t_{1}}=N_{v}\left(v\left(t_{1}\right)\right), \\
u(0)=a, \quad v(0)=b, \quad u(1)=c, \quad v(1)=d,
\end{gathered}
$$

where $F, G \in C((0,1) \times \mathbb{R} \times \mathbb{R}, \mathbb{R}), I_{u}, I_{v} \in C\left(\mathbb{R}_{+}, \mathbb{R}\right)$ satisfying $I_{u}(0)=0=I_{v}(0)$ and $N_{u}, N_{v} \in$ $C\left(\mathbb{R}_{+},(-\infty, 0]\right)$.

Definition 2.4. $\left(\alpha_{u}, \alpha_{v}\right) \in X \cap\left(C^{2}\left(J^{\prime}\right) \times C^{2}\left(J^{\prime}\right)\right)$ is called a lower solution of problem $(S)$ if

$$
\begin{gathered}
\alpha_{u}^{\prime \prime}(t)+F\left(t, \alpha_{u}(t), \alpha_{v}(t)\right) \geq 0, \quad t \neq t_{1}, \\
\alpha_{v}^{\prime \prime}(t)+G\left(t, \alpha_{u}(t), \alpha_{v}(t)\right) \geq 0, \quad t \neq t_{1}, \\
\left.\Delta \alpha_{u}\right|_{t=t_{1}}=I_{u}\left(\alpha_{u}\left(t_{1}\right)\right),\left.\quad \Delta \alpha_{v}\right|_{t=t_{1}}=I_{v}\left(\alpha_{v}\left(t_{1}\right)\right), \\
\left.\Delta \alpha_{u}^{\prime}\right|_{t=t_{1}} \geq N_{u}\left(\alpha_{u}\left(t_{1}\right)\right),\left.\quad \Delta \alpha_{v}^{\prime}\right|_{t=t_{1}} \geq N_{v}\left(\alpha_{v}\left(t_{1}\right)\right), \\
\alpha_{u}(0) \leq a, \quad \alpha_{v}(0) \leq b, \quad \alpha_{u}(1) \leq c, \quad \alpha_{v}(1) \leq d .
\end{gathered}
$$

We also define an upper solution $\left(\beta_{u}, \beta_{v}\right) \in X \cap\left(C^{2}\left(J^{\prime}\right) \times C^{2}\left(J^{\prime}\right)\right)$ if $\left(\beta_{u}, \beta_{v}\right)$ satisfies the reverses of the above inequalities. [3].

The following existence theorem for upper and lower solutions method is proved in 
Theorem 2.5. Let $\left(\alpha_{u}, \alpha_{v}\right)$ and $\left(\beta_{u}, \beta_{v}\right)$ be lower and upper solutions of problem (S), respectively, satisfying $\left(a_{1}\right)$. Moreover, we assume $\left(a_{2}\right) \sim\left(a_{4}\right)$ and $\left(D_{3}\right)$. Then problem $(S)$ has at least one solution $(u, v)$ such that

$$
\left(\alpha_{u}(t), \alpha_{v}(t)\right) \leq(u(t), v(t)) \leq\left(\beta_{u}(t), \beta_{v}(t)\right), \quad \forall t \in[0,1]
$$

The following theorems are well known cone theoretic fixed point theorems. See Lakshmikantham ([8]) for proofs and details.

Theorem 2.6. Let $X$ be a Banach space and $\mathcal{K}$ a cone in $X$. Assume that $\Omega_{1}$ and $\Omega_{2}$ are bounded open subsets in $X$ with $0 \in \Omega_{1}$ and $\Omega_{1} \subset \Omega_{2}$. Let $T: \mathcal{K} \cap\left(\bar{\Omega}_{2} \backslash \Omega_{1}\right) \rightarrow \mathcal{K}$ be a completely continuous such that either

(i) $\|T u\| \leq\|u\|$ for $u \in \mathcal{K} \cap \partial \Omega_{1}$ and $\|T u\| \geq\|u\|$ for $u \in \mathcal{K} \cap \partial \Omega_{2}$ or

(ii) $\|T u\| \geq\|u\|$ for $u \in \mathcal{K} \cap \partial \Omega_{1}$ and $\|T u\| \leq\|u\|$ for $u \in \mathcal{K} \cap \partial \Omega_{2}$.

Then $T$ has a fixed point in $\mathcal{K} \cap\left(\bar{\Omega}_{2} \backslash \Omega_{1}\right)$.

Theorem 2.7. Let $X$ be a Banach space, $\mathcal{K}$ a cone in $X$ and $\Omega$ bounded open in $X$. Let $0 \in \Omega$ and $T: \mathcal{K} \cap \bar{\Omega} \rightarrow \mathcal{K}$ be condensing. Suppose that $T x \neq v \mathcal{v}$, for all $x \in \mathcal{K} \cap \partial \Omega$ and all $v \geq 1$. Then

$$
i(T, \mathcal{K} \cap \Omega, \mathcal{K})=1 .
$$

\section{Existence}

In this section, we prove an existence theorem of positive solutions for problem $(P)$ with Dirichlet boundary condition and the existence and nonexistence part of the result for problem $(P)$ with two-point boundary condition. Let us consider the following second-order impulsive differential systems.

$$
\begin{gathered}
u^{\prime \prime}(t)+\lambda h_{1}(t) f(u(t), v(t))=0, \quad t \in(0,1), \quad t \neq t_{1}, \\
v^{\prime \prime}(t)+\mu h_{2}(t) g(u(t), v(t))=0, \quad t \in(0,1), \quad t \neq t_{1}, \\
\left.\Delta u\right|_{t=t_{1}}=I_{u}\left(u\left(t_{1}\right)\right),\left.\quad \Delta v\right|_{t=t_{1}}=I_{v}\left(v\left(t_{1}\right)\right) \\
\left.\Delta u^{\prime}\right|_{t=t_{1}}=N_{u}\left(u\left(t_{1}\right)\right),\left.\quad \Delta v^{\prime}\right|_{t=t_{1}}=N_{v}\left(v\left(t_{1}\right)\right), \\
u(0)=a \geq 0, \quad v(0)=b \geq 0, \quad u(1)=c \geq 0, \quad v(1)=d \geq 0,
\end{gathered}
$$

where $\lambda, \mu$ are positive real parameters, $f, g \in C\left(\mathbb{R}_{+}^{2},[0, \infty)\right)$ with $f(0,0)=0, g(0,0)=0$, and $f(u, v)>0, g(u, v)>0$ for all $(u, v) \neq(0,0), I_{u}, I_{v} \in C\left(\mathbb{R}_{+}, \mathbb{R}\right)$ satisfying $I_{u}(0)=0=$ $I_{v}(0), N_{u}, N_{v} \in C\left(\mathbb{R}_{+},(-\infty, 0]\right)$, and $h_{1}, h_{2} \in C((0,1),(0, \infty))$ may be singular at $t=0$ and/or 1. 
We first set up an equivalent operator equatio for problem $(P)$. Let us define $A_{\curlywedge}: X \rightarrow$ $P C[0,1]$ and $B_{\mu}: X \rightarrow P C[0,1]$ by taking

$$
\begin{aligned}
& A_{\curlywedge}(u, v)(t) \triangleq a+(c-a) t+\lambda \int_{0}^{1} K(t, s) h_{1}(s) f(u(s), v(s)) d s+W_{u}(t, u), \\
& B_{\mu}(u, v)(t) \triangleq b+(d-b) t+\mu \int_{0}^{1} K(t, s) h_{2}(s) g(u(s), v(s)) d s+W_{v}(t, v),
\end{aligned}
$$

where

$$
\begin{gathered}
K(t, s)= \begin{cases}t\left(-I_{v}\left(v\left(t_{1}\right)\right)-\left(1-t_{1}\right) N_{v}\left(v\left(t_{1}\right)\right)\right), & 0 \leq t \leq t_{1}, \\
(1-t)\left(I_{v}\left(v\left(t_{1}\right)\right)-t_{1} N_{v}\left(v\left(t_{1}\right)\right)\right), & t_{1}<t \leq 1 .\end{cases} \\
W_{u}(t, u)(t)= \begin{cases}t\left(-I_{u}\left(u\left(t_{1}\right)\right)-\left(1-t_{1}\right) N_{u}\left(u\left(t_{1}\right)\right)\right), & 0 \leq t \leq t_{1}, \\
(1-t)\left(I_{u}\left(u\left(t_{1}\right)\right)-t_{1} N_{u}\left(u\left(t_{1}\right)\right)\right), & t_{1}<t \leq 1,\end{cases} \\
W_{v}(t, u)(t)= \begin{cases}t\left(-I_{v}\left(v\left(t_{1}\right)\right)-\left(1-t_{1}\right) N_{v}\left(v\left(t_{1}\right)\right)\right), & 0 \leq t \leq t_{1}, \\
(1-t)\left(I_{v}\left(v\left(t_{1}\right)\right)-t_{1} N_{v}\left(v\left(t_{1}\right)\right)\right), & t_{1}<t \leq 1 .\end{cases}
\end{gathered}
$$

Also define

$$
T_{\lambda, \mu}(u, v) \triangleq\left(A_{\curlywedge}(u, v), B_{\mu}(u, v)\right)
$$

Then $T_{\lambda, \mu}: X \rightarrow X$ is well defined on $X$ and problem $(P)$ is equivalent to the fixed-point equation

$$
T_{\lambda, \mu}(u, v)=(u, v) \quad \text { in } X
$$

Mainly due to $\left(D_{1}\right), T_{\lambda, \mu}$ is completely continuous (see [3] for the proof). Let $\|u\|_{0}=$ $\sup _{t \in\left[0, t_{1}\right]}|u(t)|,\|u\|_{1}=\sup _{t \in\left[t_{1}, 1\right]}|u(t)|, S_{0}=\left[t_{1} / 4,3 t_{1} / 4\right], S_{1}=\left[3 t_{1}+1 / 4, t_{1}+3 / 4\right], P=\{(u, v) \in$ $X \mid u, v \geq 0\}$, and $\mathcal{K}=\left\{(u, v) \in \mathcal{D} \mid \min _{t \in S_{0}}(u(t)+v(t)) \geq t_{1} / 4\left(\|u\|_{0}+\|v\|_{0}\right), \min _{t \in S_{1}}(u(t)+\right.$ $\left.v(t)) \geq 1-t_{1} / 4\left(\|u\|_{1}+\|v\|_{1}\right)\right\}$. Then $\|u\|=\max \left\{\|u\|_{0},\|u\|_{1}\right\}$ and $\not, \mathcal{K}$ are cones in $X$. By using concavity of $T_{\lambda, \mu}(\mathrm{u})$ with $u \in \mathcal{D}$, we can easily show that $T_{\lambda, \mu}(\mathcal{D}) \subset \mathcal{K}$. 
We now prove the existence theorem of positive solutions for Dirichlet boundary value problem

$$
\begin{gathered}
u^{\prime \prime}(t)+\lambda h_{1}(t) f(u(t), v(t))=0, \quad t \in(0,1), \quad t \neq t_{1}, \\
v^{\prime \prime}(t)+\mu h_{2}(t) g(u(t), v(t))=0, \quad t \in(0,1) t \neq t_{1}, \\
\left.\Delta u\right|_{t=t_{1}}=I_{u}\left(u\left(t_{1}\right)\right),\left.\quad \Delta v\right|_{t=t_{1}}=I_{v}\left(v\left(t_{1}\right)\right), \\
\left.\Delta u^{\prime}\right|_{t=t_{1}}=N_{u}\left(u\left(t_{1}\right)\right),\left.\quad \Delta v^{\prime}\right|_{t=t_{1}}=N_{v}\left(v\left(t_{1}\right)\right), \\
u(0)=0, \quad v(0)=0, \quad u(1)=0, \quad v(1)=0 .
\end{gathered}
$$

Theorem 3.1. Assume $\left(D_{1}\right),\left(D_{2}\right),\left(D_{4}^{\prime}\right)$, and $\left(D_{5}^{\prime}\right)$. Then problem $\left(P_{D}\right)$ has at least one positive solution for all $(\lambda, \mu) \in \mathbb{R}_{+}^{2} \backslash\{(0,0)\}$.

Proof. First, we consider case $\lambda>0$ and $\mu>0$. By the fact $N_{u, 0}<1 / 2$ and $N_{v, 0}<1 / 2$, we may choose $c_{1}, m_{1}>0$ such that $\max \left\{N_{u, 0}, N_{v, 0}\right\}<c_{1}<1 / 2,\left|N_{u}(u)\right| \leq c_{1} u$ for $u \leq m_{1}$ and $\left|N_{v}(v)\right| \leq c_{1} v$ for $v \leq m_{1}$. Also choose $\eta_{\curlywedge}$ and $\eta_{\mu}$ satisfying $0<\eta_{\curlywedge}<\left(1-2 c_{1}\right) / 2 \lambda \int_{0}^{1} s(1-$ $s) h_{1}(s) d s$ and $0<\eta_{\mu}<\left(1-2 c_{1}\right) / 2 \mu \int_{0}^{1} s(1-s) h_{2}(s) d s$. Since $f_{0}=0$ and $g_{0}=0$, there exist $m_{2}, m_{3}>0$ such that $f(u, v) \leq \eta_{\lambda}(u+v)$ for $u+v \leq m_{2}$ and $g(u, v) \leq \eta_{\mu}(u+v)$ for $u+v \leq m_{3}$. Let $\Omega_{1}=B_{M_{1}}=\left\{(u, v) \in X \mid\|(u, v)\|<M_{1}\right\}$ with $M_{1}=\min \left\{m_{1}, m_{2}, m_{3}\right\}$. Then for $(u, v) \in$ $\mathcal{K} \cap \partial \Omega_{1}$, we obtain by using $\left(D_{2}\right)$

$$
\begin{aligned}
A_{\curlywedge}(u, v)(t) & =\lambda \int_{0}^{1} K(t, s) h_{1}(s) f(u(s), v(s)) d s+W_{u}(t, u) \\
& \leq \lambda \eta_{\lambda} \int_{0}^{1} s(1-s) h_{1}(s)(u(s)+v(s)) d s+\left|N_{u}\left(u\left(t_{1}\right)\right)\right| \\
& \leq\left(\lambda \eta_{\lambda} \int_{0}^{1} s(1-s) h_{1}(s) d s+c_{1}\right)\|(u, v)\| \\
& \leq \frac{1}{2}\|u, v\|
\end{aligned}
$$

for all $t \in[0,1]$. Similarly, we obtain

$$
B_{\mu}(u, v)(t) \leq \frac{1}{2\|(u, v)\|}
$$

for all $t \in[0,1]$. Thus

$$
\left\|T_{\lambda, \mu}(u, v)\right\|=\left\|A_{\curlywedge}(u, v)\right\|+\left\|B_{\mu}(u, v)\right\| \leq\|(u, v)\| .
$$


On the other hand, let us choose $\eta_{1}$ and $\eta_{2}$ such that

$$
\begin{aligned}
& \frac{1}{\eta_{2}}<\mu \min \left\{\frac{t_{1}}{8} \min _{t \in S_{0}} \int_{S_{0}} K(t, s) h_{2}(s) d s, \frac{1-t_{1}}{8} \min _{t \in S_{1}} \int_{S_{1}} K(t, s) h_{2}(s) d s\right\} . \\
& \frac{1}{\eta_{2}}<\mu \min \left\{\frac{t_{1}}{8} \min _{t \in S_{0}} \int_{S_{0}} K(t, s) h_{2}(s) d s, \frac{1-t_{1}}{8} \min _{t \in S_{1}} \int_{S_{1}} K(t, s) h_{2}(s) d s\right\} .
\end{aligned}
$$

Also by $\left(D_{5}^{\prime}\right)$, we may choose $R_{f}$ and $R_{g}$ such that $f(u, v) \geq \eta_{1}(u+v)$ for $u+v \geq R_{f}$ and $g(u, v) \geq \eta_{2}(u+v)$ for $u+v \geq R_{g}$. Let $\Omega_{2}=\left\{(u, v) \in X \mid\|(u, v)\|<M_{2}\right\}$, where $M_{2}=$ $\max \left\{8 R_{f} / t_{1}, 8 R_{f} /\left(1-t_{1}\right), 8 R_{g} / t_{1}, 8 R_{g} /\left(1-t_{1}\right), M_{1}+1\right\}$. Then $\Omega_{1} \subset \Omega_{2}$. Let $(u, v) \in \mathcal{K} \cap \partial \Omega_{2}$, then we have the following four cases: $\|u\| \geq\|v\|$ and $\|u\|=\|u\|_{0},\|u\| \geq\|v\|$ and $\|u\|=$ $\|u\|_{1},\|u\| \leq\|v\|$ and $\|v\|=\|v\|_{0}$, and $\|u\| \leq\|v\|$ and $\|v\|=\|v\|_{1}$. We consider the first case, the rest of them can be considered in a similar way. So let $\|u\| \geq\|v\|$ and $\|u\|=\|u\|_{0}$; then for $t \in S_{0}$, we have

$$
u(t)+v(t) \geq u(t) \geq \frac{t_{1}}{8}\left(2\|u\|_{0}\right) \geq \frac{t_{1}}{8}(\|u\|+\|v\|)=\frac{t_{1}}{8}\|(u, v)\| \geq R_{f} .
$$

Thus $f(u(t), v(t)) \geq \eta_{1}(u(t)+v(t))$ for $t \in S_{0}$. Since $W_{u}(t, u) \geq 0$, we get for $t \in S_{0}$,

$$
\begin{aligned}
A_{\curlywedge}(u, v)(t) & =\lambda \int_{0}^{1} K(t, s) h_{1}(s) f(u(s), v(s)) d s+W_{u}(t, u) \\
& \geq \lambda \int_{S_{0}} K(t, s) h_{1}(s) f(u(s), v(s)) d s \\
& \geq \lambda \eta_{1} \int_{S_{0}} K(t, s) h_{1}(s)(u(s)+v(s)) d s \\
& \geq \lambda \eta_{1} \frac{t_{1}}{8} \int_{S_{0}} K(t, s) h_{1}(s) d s\left(2\|u\|_{0}+2\|v\|_{0}\right) \\
& \geq \lambda \eta_{1} \frac{t_{1}}{8} \min _{t \in S_{0}} \int_{S_{0}} K(t, s) h_{1}(s) d s\|(u, v)\|>\|(u, v)\| .
\end{aligned}
$$

Therefore,

$$
\left\|T_{\lambda, \mu}(u, v)\right\| \geq\left\|A_{\curlywedge}(u, v)\right\|>\|(u, v)\|,
$$

and by Theorem 2.6, $T_{\lambda, \mu}$ has a fixed point in $\mathcal{K} \cap\left(\bar{\Omega}_{2} \backslash \Omega_{1}\right)$.

Second, consider case $\lambda>0$ and $\mu=0$. Taking $c_{1}, \eta_{\lambda}, m_{1}$, and $m_{2}$ as above and using the same computation, we may show

$$
\left\|A_{\curlywedge}(u, v)\right\| \leq \frac{1}{2}\|(u, v)\|
$$


for all $(u, v) \in \mathcal{K} \cap \partial \Omega_{1}$, where $\Omega_{1}=B_{M_{1}}$ with $M_{1}=\min \left\{m_{1}, m_{2}\right\}$. Since $\mu=0$,

$$
B_{\mu}(u, v)(\mathfrak{t})=W_{v}(t, v) \leq\left|N_{v}\left(v\left(t_{1}\right)\right)\right| \leq c_{1}\|(u, v)\| \leq \frac{1}{2}\|(u, v)\|,
$$

for all $t \in[0,1]$. Thus

$$
\left\|T_{\lambda, \mu}(u, v)\right\| \leq\left\|A_{\lambda}(u, v)\right\|+\left\|B_{\mu}(u, v)\right\| \leq\|(u, v)\|,
$$

for $(u, v) \in \mathcal{K} \cap \partial \Omega_{1}$. Now, let us choose $\eta_{1}$ and $R_{f}$ as above and let $\Omega_{2}=\{(u, v) \in X \mid$ $\left.\|(u, v)\|<M_{2}\right\}$, where $M_{2}=\max \left\{8 R_{f} / t_{1}, 8 R_{f} / 1-t_{1}, M_{1}+1\right\}$. Then $\Omega_{1} \subset \Omega_{2}$ and we can show by the same computation as above,

$$
\left\|T_{\lambda, \mu}(u, v)\right\| \geq\left\|A_{\curlywedge}(u, v)\right\|>\|(u, v)\|,
$$

for $(u, v) \in \mathcal{K} \cap \partial \Omega_{2}$ and thus $T_{\lambda, \mu}$ has a fixed point in $\mathcal{K} \cap\left(\bar{\Omega}_{2} \backslash \Omega_{1}\right)$.

Finally, consider case $\lambda=0$ and $\mu>0$. Taking $c_{1}, \eta_{\mu}, m_{1}$, and $m_{3}$ as the first case, we may show by similar argument,

$$
\left\|B_{\mu}(u, v)\right\| \leq \frac{1}{2}\|(u, v)\|, \quad\left\|A_{\lambda}(u, v)\right\| \leq \frac{1}{2}\|(u, v)\|,
$$

for all $(u, v) \in \mathcal{K} \cap \partial \Omega_{1}$, where $\Omega_{1}=B_{M_{1}}$ with $M_{1}=\min \left\{m_{1}, m_{3}\right\}$. Thus

$$
\left\|T_{\lambda, \mu}(u, v)\right\| \leq\|(u, v)\|
$$

for $(u, v) \in \mathcal{K} \cap \partial \Omega_{1}$. Now, let us choose $\eta_{2}$ and $R_{g}$ as the first case and let $\Omega_{2}=\{(u, v) \in X \mid$ $\left.\|(u, v)\|<M_{2}\right\}$, where $M_{2}=\max \left\{8 R_{g} / t_{1}, 8 R_{g} / 1-t_{1}, M_{1}+1\right\}$. Then $\Omega_{1} \subset \Omega_{2}$ and we also show similarly, as before,

$$
\left\|T_{\lambda, \mu}(u, v)\right\| \geq\left\|B_{\mu}(u, v)\right\|>\|(u, v)\|
$$

for $(u, v) \in \mathcal{K} \cap \partial \Omega_{2}$. Therefore, $T_{\lambda, \mu}$ has a fixed point in $\mathcal{K} \cap\left(\bar{\Omega}_{2} \backslash \Omega_{1}\right)$ and this completes the proof.

Now let us consider two point boundary value problems given as follows:

$$
\begin{gathered}
u^{\prime \prime}(t)+\lambda h_{1}(t) f(u(t), v(t))=0, \quad t \in(0,1), \mathrm{t} \neq t_{1}, \\
v^{\prime \prime}(t)+\mu h_{2}(t) g(u(t), v(t))=0, \quad t \in(0,1), \quad t \neq t_{1}, \\
\left.\Delta u\right|_{t=t_{1}}=I_{u}\left(u\left(t_{1}\right)\right),\left.\quad \Delta v\right|_{t=t_{1}}=I_{v}\left(v\left(t_{1}\right)\right), \\
\left.\Delta u^{\prime}\right|_{t=t_{1}}=N_{u}\left(u\left(t_{1}\right)\right),\left.\quad \Delta v^{\prime} \Delta v\right|_{t=t_{1}}=N_{v}\left(v\left(t_{1}\right)\right), \\
u(0)=a \geq 0, \quad v(0)=b \geq 0, \quad u(1)=c>a, \quad v(1)=d>b .
\end{gathered}
$$


Lemma 3.2. Assume $\left(D_{5}\right)$. Let $R$ be a compact subset of $\mathbb{R}_{+}^{2} \backslash\{(0,0)\}$. Then there exists a constant $b_{\mathcal{R}}>0$ such that for all $(\lambda, \mu) \in R$ for possible positive solutions $(u, v)$ of problem $(3.20)$ at $(\lambda, \mu)$, one has $\|(u, v)\|<b_{R}$.

Proof. Suppose on the contrary that there is a sequence $\left(u_{n}, v_{n}\right)$ of positive solutions of (3.20) at $\left(\lambda_{n}, \mu_{n}\right)$ such that $\left(\lambda_{n}, \mu_{n}\right) \in \mathcal{R}$ for all $n$ and $\left\|\left(u_{n}, v_{n}\right)\right\| \rightarrow \infty$. Since $(0,0) \notin \mathcal{R}$, there is a subsequence, say again $\left\{\left(\lambda_{n}, \mu_{n}\right)\right\}$, such that $\alpha \triangleq \min \left\{\lambda_{n}\right\}>0$ or $\beta \triangleq \min \left\{\mu_{n}\right\}>0$. First, we assume $\alpha>0$. From $\left\|\left(u_{n}, v_{n}\right)\right\| \rightarrow \infty$, we know $\left\|u_{n}\right\|_{0}+\left\|v_{n}\right\|_{0} \rightarrow \infty$ or $\left\|u_{n}\right\|_{1}+\left\|v_{n}\right\|_{1} \rightarrow \infty$. Suppose $\left\|u_{n}\right\|_{0}+\left\|v_{n}\right\|_{0} \rightarrow \infty$. Then by the concavity of $u_{n}$ and $v_{n}$, we have

$$
u_{n}(t)+v_{n}(t) \geq \frac{t_{1}}{4}\left(\left\|u_{n}\right\|_{0}+\left\|v_{n}\right\|_{0}\right)
$$

for $t \in S_{0}$. Let us choose $\eta_{1}>(2 \pi)^{2} / t_{1}^{2} \alpha \bar{h}_{1}$, where $\bar{h}_{1}=\min _{t \in S_{0}} h_{1}(t)$. Then by $\left(D_{5}\right)$, there exists $R_{f}>0$ such that

$$
f(u, v) \geq \eta_{1}(u+v) \quad \forall u+v \geq R_{f} .
$$

Since $\left\|u_{n}\right\|_{0}+\left\|v_{n}\right\|_{0}>\left(4 / t_{1}\right) R_{f}$ for sufficiently large $n$, (3.19) implies $u_{n}(t)+v_{n}(t)>R_{f}$ for $t \in S_{0}$. Thus for $t \in S_{0}$,

$$
f\left(u_{n}(t), v_{n}(t)\right)>\eta_{1}\left(u_{n}(t)+v_{n}(t)\right) \geq \eta_{1} u_{n}(t) .
$$

Hence we have for $t \in S_{0}$,

$$
0=u_{n}^{\prime \prime}(t)+\lambda_{n} h_{1}(t) f\left(u_{n}(t), v_{n}(t)\right)>u_{n}^{\prime \prime}(t)+\alpha \bar{h}_{1} \eta_{1} u_{n}(t) .
$$

If we multiply by $\phi(t)=\sin \left(2 \pi / t_{1}\right)\left(t-\left(t_{1} / 4\right)\right)$ both sides in the above inequality and integrate on $S_{0}$, then by the facts $\phi^{\prime}\left(t_{1} / 4\right)>0, \phi^{\prime}\left(3 t_{1} / 4\right)<0$ and integration by part, we obtain

$$
\begin{aligned}
& 0>\int_{t_{1} / 4}^{3 t_{1} / 4} u_{n}^{\prime \prime}(t) \phi(t) d t+\alpha \bar{h}_{1} \eta_{1} \int_{t_{1} / 4}^{3 t_{1} / 4} u_{n}(t) \phi(t) d t \\
& \geq-\left(\frac{2 \pi}{t_{1}}\right)^{2} \int_{t_{1} / 4}^{3 t_{1} / 4} u_{n}(t) \phi(t) d t+\alpha \bar{h}_{1} \eta_{1} \int_{t_{1} / 4}^{3 t_{1} / 4} u_{n}(t) \phi(t) d t .
\end{aligned}
$$

Thus $\left(2 \pi / t_{1}\right)^{2} / \alpha \bar{h}_{1} \geq \eta_{1}$ which is a contradiction to the choice of $\eta_{1}$. Suppose $\left\|u_{n}\right\|_{1}+\left\|v_{n}\right\|_{1} \rightarrow$ $\infty$, then we also get a contradiction by a similar calculation with $\eta_{2}>(2 \pi)^{2} /\left(1-t_{1}\right)^{2} \alpha \tilde{h}_{1}$, where $\tilde{h}_{1}=\min _{t \in S_{1}} h_{1}(t)$. Finally, the case $\beta>0$ can also be proved by similar way using the condition $g_{\infty}=\infty$.

Lemma 3.3. Assume $\left(D_{1}\right),\left(D_{3}\right)$, and

(Q) $f\left(u, v_{1}\right) \leq f\left(u, v_{2}\right)$, whenever $v_{1} \leq v_{2}, g\left(u_{1}, v\right) \leq g\left(u_{2}, v\right)$, whenever $u_{1} \leq u_{2}$. 
If problem (3.20) has a positive solution at $(\bar{\lambda}, \bar{\mu})$. Then the problem also has a positive solution at $(\lambda, \mu)$ for all $(\lambda, \mu) \leq(\bar{\lambda}, \bar{\mu})$.

Proof. Let $(\bar{u}, \bar{v})$ be a positive solution of problem (3.20) at $(\bar{\lambda}, \bar{\mu})$ and let $(\lambda, \mu) \in \mathbb{R}_{+}^{2} \backslash\{(0,0)\}$ with $(\lambda, \mu) \leq(\bar{\lambda}, \bar{\mu})$. Then $(\bar{u}, \bar{v})$ is an upper solution of (3.20) at $(\lambda, \mu)$. Define $\left(\alpha_{u}, \alpha_{v}\right)$ by

$$
\begin{aligned}
& \alpha_{u}(t)= \begin{cases}0, & t \in\left[0, t_{1}\right], \\
\frac{c}{1-t_{1}}\left(t-t_{1}\right), & t \in\left(t_{1}, 1\right],\end{cases} \\
& \alpha_{v}(t)= \begin{cases}0, & t \in\left[0, t_{1}\right], \\
\frac{d}{1-t_{1}}\left(t-t_{1}\right), & t \in\left(t_{1}, 1\right] .\end{cases}
\end{aligned}
$$

Then $\left(\alpha_{u}, \alpha_{v}\right)$ is a lower solution of problem (3.20) at $(\lambda, \mu)$. By the concavity of $(\bar{u}, \bar{v}),(\bar{u}, \bar{v}) \geq$ $\left(\alpha_{u}, \alpha_{v}\right)$. Therefore, Theorem 2.5 implies that problem (3.20) has a positive solution at $(\lambda, \mu)$.

Lemma 3.4. Assume $\left(D_{1}\right) \sim\left(D_{4}\right)$ and $(Q)$. Then there exists $\left(\lambda^{*}, \mu^{*}\right)>(0,0)$ such that problem (3.20) has a positive solution for all $(\lambda, \mu) \leq\left(\lambda^{*}, \mu^{*}\right)$.

Proof. It is not hard to see that the following problem:

$$
\begin{aligned}
& u^{\prime \prime}(t)+h_{1}(t)=0, t \in(0,1), \quad t \neq t_{1}, \\
& v^{\prime \prime}(t)+h_{2}(t)=0, t \in(0,1), \quad t \neq t_{1}, \\
&\left.\Delta u\right|_{t=t_{1}}=I_{u}\left(u\left(t_{1}\right)\right),\left.\Delta v\right|_{t=t_{1}}=I_{v}\left(v\left(t_{1}\right)\right), \\
&\left.\Delta u^{\prime}\right|_{t=t_{1}}=N_{u}\left(u\left(t_{1}\right)\right),\left.\Delta v^{\prime}\right|_{t=t_{1}}=N_{v}\left(v\left(t_{1}\right)\right), \\
& u(0)=a \geq 0, \quad v(0)=b \geq 0, \quad u(1)=c>a, \quad v(1)=d>b
\end{aligned}
$$

has a positive solution so let $\left(\beta_{u}, \beta_{v}\right)$ be a positive solution. Let $M_{f}=\sup _{t \in[0,1]} f\left(\beta_{u}(t), \beta_{v}(t)\right)$ and $M_{g}=\sup _{t \in[0,1]} g\left(\beta_{u}(t), \beta_{v}(t)\right)$. Then $M_{f}, M_{g}>0$ and for $\left(\lambda^{*}, \mu^{*}\right)=\left(1 / M_{f}, 1 / M_{g}\right)$, we get

$$
\begin{aligned}
& \beta_{u}^{\prime \prime}+\lambda^{*} h_{1}(t) f\left(\beta_{u}(t), \beta_{v}(t)\right)=h_{1}(t)\left(\lambda^{*} f\left(\beta_{u}(t), \beta_{v}(t)\right)-1\right) \leq 0, \\
& \beta_{v}^{\prime \prime}+\mu^{*} h_{2}(t) g\left(\beta_{u}(t), \beta_{v}(t)\right)=h_{2}(t)\left(\mu^{*} g\left(\beta_{u}(t), \beta_{v}(t)\right)-1\right) \leq 0 .
\end{aligned}
$$

This shows that $\left(\beta_{u}, \beta_{v}\right)$ is an upper solution of (3.20) at $\left(\lambda^{*}, \mu^{*}\right)$. On the other hand, $\left(\alpha_{u}, \alpha_{v}\right)$ given in Lemma 3.3 is obviously a lower solution and $\left(\alpha_{u}, \alpha_{v}\right) \leq\left(\beta_{u}, \beta_{v}\right)$. Thus by Theorem 2.5, (3.20) has a positive solution at $\left(\lambda^{*}, \mu^{*}\right)$ and the proof is done by Lemma 3.3.

We introduce a known existence result for a singular boundary value problem with no impulse effect. 
Lemma 3.5 (see[9]). Consider, $\left(D_{1}\right),\left(D_{5}\right)$ and $(Q)$. For problem

$$
\begin{gathered}
u^{\prime \prime}(t)+\lambda h_{1}(t) f(u(t), v(t))=0, \\
v^{\prime \prime}(t)+\mu h_{2}(t) g(u(t), v(t))=0, \quad t \in(0,1), \\
u(0)=a \geq 0, \quad u(1)=c>a, \\
v(0)=b \geq 0, \quad v(1)=d>b,
\end{gathered}
$$

let $\mathscr{A}_{T}=\left\{(\lambda, \mu) \in \mathbb{R}_{+}^{2} \backslash\{(0,0)\} \mid(3.27)\right.$ has a positive solution at $\left.(\lambda, \mu)\right\}$. Then $\left(\mathcal{A}_{T}, \leq\right)$ is bounded above.

Define $\mathcal{A}=\left\{(\lambda, \mu) \in \mathbb{R}_{+}^{2} \backslash\{(0,0)\} \mid(3.20)\right.$ has a positive solution at $\left.(\lambda, \mu)\right\}$. Then $\mathcal{A} \neq \emptyset$ by Lemma 3.4 and $(\mathcal{A}, \leq)$ is a partially ordered set.

Lemma 3.6. Assume $\left(D_{1}\right) \sim\left(D_{6}\right)$. Then $(\mathcal{A}, \leq)$ is bounded above.

Proof. Suppose on the contrary that there exists a sequence $\left(\lambda_{n}, \mu_{n}\right) \in \mathscr{A}$ such that $\left|\left(\lambda_{n}, \mu_{n}\right)\right| \rightarrow$ $\infty$. Let $\left(u_{n}, v_{n}\right)$ be a positive solution of problem (3.20) at $\left(\lambda_{n}, \mu_{n}\right)$. By condition $\left(D_{2}\right)$, we may choose sequences $\left(s_{n}\right),\left(t_{n}\right)$ in $\left[0, t_{1}\right) \cup\left(t_{1}, 1\right]$ such that if $I_{u}\left(u_{n}\left(t_{1}\right)\right)>0$, then $t_{n} \in\left(t_{1}, 1\right]$ and

$$
\begin{aligned}
& I_{u}\left(u_{n}\left(t_{1}\right)\right)+\left(t_{n}-t_{1}\right) N_{u}\left(u_{n}\left(t_{1}\right)\right)=0, \\
& I_{u}\left(u_{n}\left(t_{1}\right)\right)+\left(t-t_{1}\right) N_{u}\left(u_{n}\left(t_{1}\right)\right)>0, \quad \text { on }\left[t_{1}, t_{n}\right) \text {, } \\
& I_{u}\left(u_{n}\left(t_{1}\right)\right)+\left(t-t_{1}\right) N_{u}\left(u_{n}\left(t_{1}\right)\right)<0, \quad \text { on }\left(t_{n}, 1\right] \text {; }
\end{aligned}
$$

if $I_{u}\left(u_{n}\left(t_{1}\right)\right)<0$, then $t_{n} \in\left[0, t_{1}\right)$ and

$$
\begin{aligned}
& I_{u}\left(u_{n}\left(t_{1}\right)\right)+\left(t_{n}-t_{1}\right) N_{u}\left(u_{n}\left(t_{1}\right)\right)=0, \\
& I_{u}\left(u_{n}\left(t_{1}\right)\right)+\left(t-t_{1}\right) N_{u}\left(u_{n}\left(t_{1}\right)\right)>0, \quad \text { on }\left[0, t_{n}\right) \text {, } \\
& I_{u}\left(u_{n}\left(t_{1}\right)\right)+\left(t-t_{1}\right) N_{u}\left(u_{n}\left(t_{1}\right)\right)<0, \quad \text { on }\left(t_{n}, t_{1}\right] \text {; }
\end{aligned}
$$

if $I_{v}\left(v_{n}\left(t_{1}\right)\right)>0$, then $s_{\mathrm{n}} \in\left(t_{1}, 1\right]$ and

$$
\begin{aligned}
& I_{v}\left(v_{n}\left(t_{1}\right)\right)+\left(s_{n}-t_{1}\right) N_{v}\left(v_{n}\left(t_{1}\right)\right)=0, \\
& I_{v}\left(v_{n}\left(t_{1}\right)\right)+\left(t-t_{1}\right) N_{v}\left(v_{n}\left(t_{1}\right)\right)>0, \quad \text { on }\left[t_{1}, s_{n}\right) \text {, } \\
& I_{v}\left(v_{n}\left(t_{1}\right)\right)+\left(t-t_{1}\right) N_{v}\left(v_{n}\left(t_{1}\right)\right)<0, \quad \text { on }\left(s_{n}, 1\right] \text {; }
\end{aligned}
$$

if $I_{v}\left(v_{n}\left(t_{1}\right)\right)<0$, then $s_{n} \in\left[0, t_{1}\right)$ and

$$
\begin{gathered}
I_{v}\left(v_{n}\left(t_{1}\right)\right)+\left(s_{n}-t_{1}\right) N_{v}\left(v_{n}\left(t_{1}\right)\right)=0, \\
I_{v}\left(v_{n}\left(t_{1}\right)\right)+\left(t-t_{1}\right) N_{v}\left(v_{n}\left(t_{1}\right)\right)>0, \quad \text { on }\left[0, s_{n}\right), \\
I_{v}\left(v_{n}\left(t_{1}\right)\right)+\left(t-t_{1}\right) N_{v}\left(v_{n}\left(t_{1}\right)\right)<0, \quad \text { on }\left(s_{n}, t_{1}\right] .
\end{gathered}
$$


If $I_{u}\left(u_{n}\left(t_{1}\right)\right)>0$, define

$$
\tilde{u}_{n}(t)= \begin{cases}u_{n}(t), & \text { on }\left[0, t_{1}\right] \\ u_{n}(t)-\left(I_{u}\left(u_{n}\left(t_{1}\right)\right)+\left(t-t_{1}\right) N_{u}\left(u_{n}\left(t_{1}\right)\right)\right), & \text { on }\left(t_{1}, t_{n}\right) \\ u_{n}(t), & \text { on }\left[t_{n}, 1\right]\end{cases}
$$

and if $I_{u}\left(u_{n}\left(t_{1}\right)\right)<0$, define

$$
\tilde{u}_{n}(t)= \begin{cases}u_{n}(t), & \text { on }\left[0, t_{n}\right] \\ u_{n}(t)+\left(I_{u}\left(u_{n}\left(t_{1}\right)\right)+\left(t-t_{1}\right) N_{u}\left(u_{n}\left(t_{1}\right)\right)\right), & \text { on }\left(t_{n}, t_{1}\right] \\ u_{n}(t), & \text { on }\left(t_{1}, 1\right]\end{cases}
$$

Moreover, if $I_{v}\left(v_{n}\left(t_{1}\right)\right)>0$, define

$$
\tilde{v}_{n}(t)= \begin{cases}v_{n}(t), & \text { on }\left[0, t_{1}\right], \\ v_{n}(t)-\left(I_{v}\left(v_{n}\left(t_{1}\right)\right)+\left(t-t_{1}\right) N_{v}\left(v_{n}\left(t_{1}\right)\right)\right), & \text { on }\left(t_{1}, s_{n}\right), \\ v_{n}(t), & \text { on }\left[s_{n}, 1\right]\end{cases}
$$

and if $I_{v}\left(v_{n}\left(t_{1}\right)\right)<0$, define

$$
\tilde{v}_{n}(t)= \begin{cases}v_{n}(t), & \text { on }\left[0, s_{n}\right] \\ v_{n}(t)+\left(I_{v}\left(v_{n}\left(t_{1}\right)\right)+\left(t-t_{1}\right) N_{v}\left(v_{n}\left(t_{1}\right)\right)\right), & \text { on }\left(s_{n}, t_{1}\right] \\ v_{n}(t), & \text { on }\left(t_{1}, 1\right]\end{cases}
$$

Then we can easily see that $\left(\tilde{u}_{n}, \widetilde{v}_{n}\right) \in(C[0,1] \times C[0,1]) \cap\left(C^{2}(0,1) \times C^{2}(0,1)\right)$ except $t_{1}, t_{n}, s_{n}$. Furthermore, $\left(\tilde{u}_{n}^{\prime}\left(t_{1}^{-}\right), \widetilde{v}_{n}^{\prime}\left(t_{1}^{-}\right)\right)=\left(\tilde{u}_{n}^{\prime}\left(t_{1}^{+}\right), \widetilde{v}_{n}^{\prime}\left(t_{1}^{+}\right)\right),\left(\tilde{u}_{n}^{\prime}\left(t_{n}^{-}\right), \widetilde{v}_{n}^{\prime}\left(t_{n}^{-}\right)\right) \geq\left(\tilde{u}_{n}^{\prime}\left(t_{n}^{+}\right), \widetilde{v}_{n}^{\prime}\left(t_{n}^{+}\right)\right)$and $\left(\tilde{u}_{n}^{\prime}\left(s_{n}^{-}\right), \tilde{v}_{n}^{\prime}\left(s_{n}^{-}\right)\right) \geq\left(\tilde{u}_{n}^{\prime}\left(s_{n}^{+}\right), \widetilde{v}_{n}^{\prime}\left(s_{n}^{+}\right)\right)$. We also see $\left(u_{n}(t), v_{n}(t)\right) \geq\left(\tilde{u}_{n}(t), \tilde{v}_{n}(t)\right)$ on $[0,1]$. Thus by $\left(D_{6}\right)$, we get

$$
\begin{aligned}
\tilde{u}_{n}^{\prime \prime}(t)+\lambda_{n} h_{1}(t) f\left(\tilde{u}_{n}(t), \tilde{v}_{n}(t)\right) & =u_{n}^{\prime \prime}(t)+\lambda_{n} h_{1}(t) f\left(\tilde{u}_{n}(t), \tilde{v}_{n}(t)\right) \\
& =\lambda_{n} h_{1}(t)\left(f\left(\tilde{u}_{n}(t), \tilde{v}_{n}(t)\right)-f\left(u_{n}(t), v_{n}(t)\right)\right) \leq 0, \\
\tilde{v}_{n}^{\prime \prime}(t)+\mu_{n} h_{2}(t) g\left(\tilde{u}_{n}(t), \tilde{v}_{n}(t)\right) & =v_{n}^{\prime \prime}(t)+\mu_{n} h_{2}(t) g\left(\tilde{u}_{n}(t), \tilde{v}_{n}(t)\right) \\
& =\mu_{n} h_{2}(t)\left(g\left(\tilde{u}_{n}(t), \tilde{v}_{n}(t)\right)-g\left(u_{n}(t), v_{n}(t)\right)\right) \leq 0 .
\end{aligned}
$$

We also get $\tilde{u}_{n}(0)=u_{n}(0)=a, \tilde{u}_{n}(1)=u_{n}(1)=c, \widetilde{v}_{n}(0)=v_{n}(0)=b$, and $\tilde{v}_{n}(1)=v_{n}(1)=d$. Thus $\left(\tilde{u}_{n}, \tilde{v}_{n}\right)$ is a $G$-upper solution of problem $\left(U_{T}\right)$ at $\left(\lambda_{n}, \mu_{n}\right)$. If $I_{u}\left(u_{n}\left(t_{1}\right)\right)=0$ or $I_{v}\left(v_{n}\left(t_{1}\right)\right)=$ 0 , then we consider $\widetilde{u}_{n}=u_{n}$ or $\widetilde{v}_{n}=v_{n}$ as a G-upper solution. Let $\left(\widetilde{\alpha}_{u}(t), \tilde{\alpha}_{v}(t)\right)=((c-$ $a) t+a,(d-b) t+b)$, then $\left(\tilde{\alpha}_{u}, \tilde{\alpha}_{v}\right)$ is the G-lower solution of (3.27) at $\left(\lambda_{n}, \mu_{n}\right)$. Therefore, 
by Theorem 2.3, problem (3.27) has a positive solution for all $\left(\lambda_{n}, \mu_{n}\right)$. This contradicts to Lemma 3.5 and the proof is done.

Lemma 3.7. Assume $\left(D_{1}\right) \sim\left(D_{6}\right)$. Then every nonempty chain in $\mathbb{A}$ has a unique supremum in $\mathcal{A}$.

Proof. Let $\mathcal{C}$ be a chain in $\mathcal{A}$. Without loss of generality, we may choose a distinct sequence $\left\{\left(\lambda_{n}, \mu_{n}\right)\right\} \subset \mathcal{C}$ such that $\left(\lambda_{n}, \mu_{n}\right) \leq\left(\lambda_{n+1}, \mu_{n+1}\right)$. By Lemma 3.6, there exists $\left(\lambda_{C}, \mu_{C}\right)$ such that $\left(\lambda_{n}, \mu_{n}\right) \rightarrow\left(\lambda_{C}, \mu_{C}\right)$. If we show $\left(\lambda_{C}, \mu_{C}\right) \in \mathcal{A}$, then the proof is done. Since $\left\{\left(\lambda_{n}, \mu_{n}\right)\right\}$ is bounded, Lemma 3.2 implies that there is a constant $B$ such that $\left\|\left(u_{n}, v_{n}\right)\right\|<B$, where $\left(u_{n}, v_{n}\right)$ is a solution corresponding to $\left(\lambda_{n}, \mu_{n}\right)$. By the compactness of $T_{\lambda, \mu},\left\{\left(u_{n}, v_{n}\right)\right\}$ has a convergent subsequence converging to say, $\left(u_{\mathcal{C}}, v_{\mathcal{C}}\right)$. By Lebesgue Convergence theorem, we see that $\left(u_{\mathcal{C}}, v_{\mathcal{C}}\right)$ is a solution of $(3.20)$ at $\left(\lambda_{\mathcal{C}}, \mu_{\mathcal{C}}\right)$. Thus $\left(\lambda_{\mathcal{C}}, \mu_{\mathcal{C}}\right) \in \mathcal{A}$.

Theorem 3.8. Assume $\left(D_{1}\right) \sim\left(D_{6}\right.$.) Then there exists a continuous curve $\Gamma$ splitting $\mathbb{R}_{+}^{2} \backslash\{(0,0)\}$ into two disjoint subsets $\mathcal{O}_{1}$ and $\mathcal{O}_{2}$ such that problem $\left(P_{T}\right)$ has at least one positive solution for $(\lambda, \mu) \in \mathcal{O}_{1} \cup \Gamma$ and no solution for $(\lambda, \mu) \in \mathcal{O}_{2}$.

Proof. $\left(\lambda^{*}, \mu^{*}\right)$ is given in Lemma 3.4. We know from Lemma 3.4 that (3.20) has a positive solution at $(0, s)$ for all $0<s \leq \mu^{*}$. Thus $\{(0, s) \mid s>0\} \cap \mathbb{A}$ is a nonempty chain in $\mathcal{A}$ and by Lemma 3.7, it has unique supremum of the form $\left(0, s^{*}\right)$ in $\mathcal{A}$. This implies that (3.20) has a positive solution at $(0, s)$ for all $0<s \leq s^{*}$ and no solution at $(0, s)$ for all $s>s^{*}$. Similarly, there is $r^{*} \geq \lambda^{*}$ such that (3.20) has a positive solution at $(r, 0)$ for all $0<r \leq r^{*}$ and no solution at $(r, 0)$ for all $r>r^{*}$. Define $L: \mathbb{R} \rightarrow \mathbb{R}^{2}$ by taking $L(t)=\{(r, s) \mid s=r+t\}$. Then for $t \in\left[-r^{*}, s^{*}\right], L(t) \cap \mathscr{A}$ is a nonempty chain in $\mathcal{A}$. Define $\Gamma(t)$ as the unique supremum of $L(t) \cap \mathcal{A}$. Then $\Gamma$ is well defined on $\left[-r^{*}, s^{*}\right]$ and as a consequence of Lemma 3.3, we see that $\Gamma$ is continuous on $\left[-r^{*}, s^{*}\right], \Gamma\left(-r^{*}\right)=\left(r^{*}, 0\right)$, and $\Gamma\left(s^{*}\right)=\left(0, s^{*}\right)$. Therefore, the curve $\Gamma=\Gamma\left[-r^{*}, s^{*}\right]$ separates $\mathbb{R}_{+}^{2} \backslash\{(0,0)\}$ into two disjoint subsets $\mathcal{O}_{1}$ and $\mathcal{O}_{2}$, where $\mathcal{O}_{1}$ is bounded and $\mathcal{O}_{2}$ is unbounded and we get the conclusion of this theorem for $\Gamma, \mathcal{O}_{1}$, and $\mathcal{O}_{2}$.

\section{Multiplicity}

In this section, we study existence of the second positive solution for two point boundary value problem (3.20) with $(\lambda, \mu)$ in certain region of $\mathcal{O}_{1}$ appeared in Theorem 3.8. For the computation of fixed point index, we need to consider problems of the form

$$
\begin{gathered}
u^{\prime \prime}(t)+\lambda h_{1}(t) f(u(t), v(t))=0, \quad t \in(0,1), \quad t \neq t_{1}, \\
v^{\prime \prime}(t)+\mu h_{2}(t) g(u(t), v(t))=0, \quad t \in(0,1), \quad t \neq t_{1}, \\
\left.\Delta u\right|_{t=t_{1}}=I_{u}\left(u\left(t_{1}\right)\right),\left.\quad \Delta v\right|_{t=t_{1}}=I_{v}\left(v\left(t_{1}\right)\right), \\
\left.\Delta u^{\prime}\right|_{t=t_{1}}=N_{u}\left(u\left(t_{1}\right)\right),\left.\quad \Delta v^{\prime}\right|_{t=t_{1}}=N_{v}\left(v\left(t_{1}\right)\right), \\
u(0)=a+\varepsilon, \quad u(1)=c+\varepsilon, \\
v(0)=b+\varepsilon, \quad v(1)=d+\varepsilon,
\end{gathered}
$$

where $\varepsilon>0, c>a \geq 0$ and $d>b \geq 0$. Theorem 3.8 implies that there exists a continuous curve $\Gamma_{\varepsilon}$ splitting $\mathbb{R}_{+}^{2} \backslash\{(0,0)\}$ into two disjoint subsets $\mathcal{O}_{\varepsilon, 1}$ and $\mathcal{O}_{\varepsilon, 2}$ such that the problem (4.1) has at least one positive solution for $(\lambda, \mu) \in \mathcal{O}_{\varepsilon, 1} \cup \Gamma_{\varepsilon}$ and no solution for $(\lambda, \mu) \in \mathcal{O}_{\varepsilon, 2}$. Using upper 
and lower solutions argument, we can easily show that if $0<\bar{\varepsilon}<\varepsilon$, then $\mathcal{O}_{\varepsilon, 1} \cup \Gamma_{\varepsilon} \subset \mathcal{O}_{\bar{\varepsilon}, 1} \cup \Gamma_{\bar{\varepsilon}}$. Let $\mathcal{O}=\cup_{\varepsilon>0}\left(\mathcal{O}_{\varepsilon, 1} \cup \Gamma_{\varepsilon}\right)$, then $\mathcal{O} \subset \mathcal{O}_{0,1}$. We state the main theorem for two point boundary value problem (3.20) as follows.

Theorem 4.1. Assume $\left(D_{1}\right) \sim\left(D_{6}\right)$. Then there exists a continuous curve $\Gamma_{0}$ splitting $\mathbb{R}_{+}^{2} \backslash\{(0,0)\}$ into two disjoint subsets $\mathcal{O}_{0,1}$ and, $\mathcal{O}_{0,2}$ and there exists a subset $\mathcal{O} \subset \mathcal{O}_{0,1}$ such that problem (3.20) has at least two positive solutions for $(\lambda, \mu) \in \mathcal{O}$, at least one positive solution for $(\lambda, \mu) \in\left(\mathcal{O}_{0,1} \backslash \mathcal{O}\right) \cup \Gamma_{0}$, and no solution for $(\lambda, \mu) \in \mathcal{O}_{0,2}$.

Proof. Let $\mathcal{O}=\cup_{\varepsilon>0}\left(\mathcal{O}_{\varepsilon, 1} \cup \Gamma_{\varepsilon}\right)$ and let $(\lambda, \mu) \in \mathcal{O}$. It is enough to prove that problem (3.20) has the second solution at $(\lambda, \mu)$. By the definition of $\mathcal{O}$, there exists $\varepsilon>0$ such that $(\lambda, \mu) \in \mathcal{O}_{\varepsilon, 1} \cup \Gamma_{\varepsilon}$. That is (4.1) has a positive solution at $(\lambda, \mu)$. So let $\left(u_{\varepsilon}, v_{\varepsilon}\right)$ be a positive solution of problem (4.1) at $(\lambda, \mu)$ and let $\Omega=\left\{(u, v) \in X \mid-\varepsilon<u(t)<u_{\varepsilon}(t),-\varepsilon<v(t)<v_{\varepsilon}(t)\right.$ for $t \in[0,1], u\left(t_{1}^{+}\right)<$ $\left.u_{\varepsilon}\left(t_{1}^{+}\right), v\left(t_{1}^{+}\right)<v_{\varepsilon}\left(t_{1}^{+}\right)\right\}$. Then $\Omega$ is bounded open in $X, 0 \in \Omega$. Furthermore, $T_{\lambda, \mu}: \mathcal{K} \cap \bar{\Omega} \rightarrow$ $\mathcal{K}$ is condensing, since it is completely continuous. We show that $T_{\lambda, \mu}(u, v) \neq v(u, v)$ for all $(u, v) \in \mathcal{K} \cap \partial \Omega$ and all $v \geq 1$. If it is not true, then there exist $(u, v) \in \mathcal{K} \cap \partial \Omega$ and $v_{0} \geq 1$ such that $T_{\lambda, \mu}(u, v)=v_{0}(u, v)$. Thus $(u, v)$ is a positive solution of the following equation

$$
\begin{gathered}
v_{0} u^{\prime \prime}(t)+\lambda h_{1}(t) f(u(t), v(t))=0, \quad t \in(0,1), \quad t \neq t_{1}, \\
v_{0} v^{\prime \prime}(t)+\mu h_{2}(t) g(u(t), v(t))=0, \quad t \in(0,1), \quad t \neq t_{1}, \\
\left.v_{0} \Delta u\right|_{t=t_{1}}=I_{u}\left(u\left(t_{1}\right)\right),\left.\quad v_{0} \Delta v\right|_{t=t_{1}}=I_{v}\left(v\left(t_{1}\right)\right), \\
\left.v_{0} \Delta u^{\prime}\right|_{t=t_{1}}=N_{u}\left(u\left(t_{1}\right)\right),\left.\quad v_{0} \Delta v^{\prime}\right|_{t=t_{1}}=N_{v}\left(v\left(t_{1}\right)\right), \\
v_{0} u(0)=a, \quad v_{0} v(0)=b, \quad v_{0} u(1)=c, \quad v_{0} v(1)=d,
\end{gathered}
$$

and we can consider the following two cases. The first case is $u\left(t_{0}\right)=u_{\varepsilon}\left(t_{0}\right)$ or $v\left(t_{0}\right)=v_{\varepsilon}\left(t_{0}\right)$ for some $t_{0} \in(0,1)$. The second case is $u\left(t_{1}^{+}\right)=u_{\varepsilon}\left(\mathrm{t}_{1}^{+}\right)$or $v\left(t_{1}^{+}\right)=v_{\varepsilon}\left(t_{1}^{+}\right)$. First, let us consider case $u\left(t_{0}\right)=u_{\varepsilon}\left(t_{0}\right)$ for some $t_{0} \in(0,1)$. One may prove similarly for case $v\left(t_{0}\right)=v_{\varepsilon}\left(t_{0}\right)$. If $t_{0} \in J^{\prime}$, that is, $t_{0} \neq t_{1}$, let $m(t)=\left(u-u_{\varepsilon}\right)(t)$, then $m^{\prime \prime}(t) \geq 0$ on $J^{\prime}, m(0)<0, m(1)<0$, and $m\left(t_{1}\right) \leq 0$. Thus on one of intervals $\left(0, t_{1}\right)$ or $\left(t_{1}, 1\right)$ containing $t_{0}$, maximum principle implies $m \equiv 0$ and this contradicts to the facts of $m(0)<0$ and $m(1)<0$. If $t_{0}=t_{1}$, then $u\left(t_{1}\right)=u_{\varepsilon}\left(t_{1}\right), u(t) \leq u_{\varepsilon}(t)$ and $v(t) \leq v_{\varepsilon}(t)$ on $[0,1]$. Thus by $\left(D_{6}\right)$ and $\left(D_{2}\right)$, we get the following contradiction:

$$
\begin{aligned}
u\left(t_{1}\right)=\frac{1}{v_{0}} A_{\curlywedge}(u, v)\left(t_{1}\right)= & \frac{a+(b-a) t_{1}}{v_{0}}+\frac{\lambda}{v_{0}} \int_{0}^{1} K\left(t_{1}, s\right) h_{1}(s) f(u(s), v(s)) d s \\
& +\frac{-t_{1}\left(I_{u}\left(u\left(t_{1}\right)\right)+\left(1-t_{1}\right) N_{u}\left(u\left(t_{1}\right)\right)\right)}{v_{0}} \\
< & a+\varepsilon+(b-a) t_{1}+\lambda \int_{0}^{1} K\left(t_{1}, s\right) h_{1}(s) f\left(u_{\varepsilon}(s), v_{\varepsilon}(s)\right) d s \\
& -t_{1}\left(I_{u}\left(u_{\varepsilon}\left(t_{1}\right)\right)+\left(1-t_{1}\right) N_{u}\left(u_{\varepsilon}\left(t_{1}\right)\right)\right) \\
= & u_{\varepsilon}\left(t_{1}\right)=u\left(t_{1}\right) .
\end{aligned}
$$


Second, let us consider $u\left(t_{1}^{+}\right)=u_{\varepsilon}\left(t_{1}^{+}\right)$. Since $u(t) \leq u_{\epsilon}(t)$ and $v(t) \leq v_{\varepsilon}(t)$, we get

$$
\begin{aligned}
u\left(t_{1}^{+}\right)=\frac{1}{v_{0}} A_{\curlywedge}(u, v)\left(t_{1}^{+}\right)= & \frac{a+(b-a) t_{1}}{v_{0}}+\frac{\lambda}{v_{0}} \int_{0}^{1} K\left(t_{1}, s\right) h_{1}(s) f(u(s), v(s)) d s \\
& +\frac{\left(1-t_{1}\right)\left(I_{u}\left(u\left(t_{1}\right)\right)-t_{1} N_{u}\left(u\left(t_{1}\right)\right)\right)}{v_{0}} \\
< & a+\varepsilon+(b-a) t_{1}+\lambda \int_{0}^{1} K\left(t_{1}, s\right) h_{1}(s) f\left(u_{\varepsilon}(s), v_{\varepsilon}(s)\right) d s \\
& +\left(1-t_{1}\right)\left(I_{u}\left(u_{\varepsilon}\left(t_{1}\right)\right)-t_{1} N_{u}\left(u_{\varepsilon}\left(t_{1}\right)\right)\right) \\
= & u_{\varepsilon}\left(t_{1}^{+}\right)=u\left(t_{1}^{+}\right) .
\end{aligned}
$$

One may show the contradiction similarly for case $v\left(t_{1}^{+}\right)=v_{\varepsilon}\left(t_{1}^{+}\right)$. This contradiction shows $T_{\lambda, \mu}(u, v) \neq v(u, v)$ for all $(u, v) \in \mathcal{K} \cap \partial \Omega$ and $v \geq 1$. Therefore, by Theorem 2.7, we obtain

$$
i\left(T_{\lambda, \mu}, \mathcal{K} \cap \Omega, \mathcal{K}\right)=1
$$

On the other hand, by Lemma 3.6, we know that there is $\left(\lambda_{1}, \mu_{1}\right)$ such that (3.20) has no positive solution at $\left(\lambda_{1}, \mu_{1}\right)$.Thus for any open set $\mathcal{U}$ in $X$, we get

$$
i\left(T_{\lambda_{1}, \mu_{1}}, \mathcal{K} \cap \mathcal{U}, \mathcal{K}\right)=0
$$

Let $\mathcal{R}$ be a compact rectangle containing $(\lambda, \mu)$ and $\left(\lambda_{1}, \mu_{1}\right)$. By Lemma 3.2, for all $(\bar{\lambda}, \bar{\mu}) \in \mathcal{R}$, there exists $b_{\mathcal{R}}>0$ such that all possible solutions $(u, v)$ of $\left(P_{T}\right)$ at $(\bar{\lambda}, \bar{\mu})$ satisfy $\|(u, v)\|<b_{\mathcal{R}}$ and $\Omega \subset B_{b_{R}}$. Define $h:[0,1] \times\left(\bar{B}_{b_{R}} \cap \mathcal{K}\right) \rightarrow \mathcal{K}$ by

$$
h(\tau,(u, v))=T_{\tau \lambda_{1}+(1-\tau) \lambda, \tau \mu_{1}+(1-\tau) \mu}(u, v) .
$$

Then $h(0,(u, v))=T_{\lambda, \mu}(u, v), h(1,(u, v))=T_{\lambda_{1}, \mu_{1}}(u, v), h$ is completely continuous on $[0,1] \times$ $\mathcal{K}$, and $h(\tau,(u, v)) \neq(u, v)$ for all $(\tau,(u, v)) \in[0,1] \times\left(\partial B_{b_{R}} \cap \mathcal{K}\right)$. By the property of homotopy invariance and (4.5), we have

$$
i\left(T_{\lambda, \mu}, B_{b_{R}} \cap \mathcal{K}, \mathcal{K}\right)=i\left(T_{\lambda_{1}, \mu_{1}}, B_{b_{\mathcal{R}}} \cap \mathcal{K}, \mathcal{K}\right)=0
$$

By the additive property and (4.4), (4.7), we have

$$
i\left(T_{\lambda, \mu},\left(\frac{B_{b_{R}}}{\bar{\Omega}}\right) \cap \mathcal{K}, \mathcal{K}\right)=-1
$$

Therefore, (3.20) has another positive solution in $\left(B_{b_{R}} \backslash \bar{\Omega}\right) \cap \mathcal{K}$ at $(\lambda, \mu) \in \mathcal{O}$ and this completes the proof. 


\section{Applications}

In this section, we apply the results in previous sections to study the existence and multiplicity theorems of positive radial solutions for impulsive semilinear elliptic problems.

\subsection{On an Annular Domain}

Let us consider

$$
\begin{gathered}
\Delta u+\lambda k_{1}(|x|) f(u, v)=0, \\
\Delta v+\mu k_{2}(|x|) g(u, v)=0, \quad \text { in } \Omega\left(l_{1}, l_{2}\right),|x| \neq r_{1}, \\
\left.\Delta u\right|_{|x|=r_{1}}=I_{u}\left(\left.u\right|_{|x|=r_{1}}\right),\left.\quad \Delta v\right|_{|x|=r_{1}}=I_{v}\left(\left.u\right|_{|x|=r_{1}}\right), \\
\left.\frac{\Delta \partial u}{\partial r}\right|_{|x|=r_{1}}=-\frac{r_{1}^{1-n} N_{u}\left(\left.u\right|_{|x|=r_{1}}\right)}{m},\left.\quad \frac{\Delta \partial v}{\partial r}\right|_{|x|=r_{1}}=-\frac{r_{1}^{1-n} N_{v}\left(\left.v\right|_{|x|=r_{1}}\right)}{m}, \\
u(x)=a \geq 0, \quad v(x)=b \geq 0 \quad \text { if }|x|=l_{1}, \\
u(x)=c>a, \quad v(x)=d>b \quad \text { if }|x|=l_{2},
\end{gathered}
$$

where $f(0,0)=0, g(0,0)=0, \Delta$ is the Laplacian of $u, 0<l_{1}<r_{1}<l_{2}$, and $\Omega\left(l_{1}, l_{2}\right)=\{x \in$ $\left.\mathbf{R}^{n}\left|l_{1}<\right| x \mid<l_{2}\right\}$ with $n>2 . \partial u / \partial r$ denotes the differentiation in the radial direction, $\left.\Delta u\right|_{|x|=r_{1}}=u\left(r_{1}^{+}\right)-u\left(r_{1}\right),\left.\Delta(\partial u / \partial r)\right|_{|x|=r_{1}}=(\partial u / \partial r)\left(r_{1}^{+}\right)-(\partial u / \partial r)\left(r_{1}^{-}\right)$and $m=-\int_{l_{1}}^{l_{2}} t^{1-n} d t$. Applying consecutive changes of variables, $r=|x|, s=-\int_{r}^{l_{2}} t^{1-n} d t$ and $t=m-s / m$, we may transform problem (5.1) into problem (3.20), where $t_{1}=\left(r_{1}^{2-n}-l_{1}^{2-n}\right) /\left(l_{2}^{2-n}-l_{1}^{2-n}\right)$ and $h_{i}$ can be written as

$$
h_{i}(t)=m^{2}[r(m(1-t))]^{2(n-1)} k_{i}(r(m(1-t))) .
$$

If $k_{i}:\left[l_{1}, l_{2}\right] \rightarrow(0, \infty)$ are continuous, then $h_{i}:[0,1] \rightarrow(0, \infty)$ are also continuous and satisfies $\left(D_{1}\right)$. We may apply Theorem 4.1 to obtain the following result.

Corollary 5.1. Assume $\left(D_{2}\right) \sim\left(D_{6}\right)$. Let $k_{i} \in C\left(\left[l_{1}, l_{2}\right],(0, \infty)\right), i=1,2$. Then there exists a continuous curve $\Gamma_{0}$ splitting $\mathbb{R}_{+}^{2} \backslash\{(0,0)\}$ into two disjoint subsets $\mathcal{O}_{0,1}$ and $\mathcal{O}_{0,2}$ and there exists a subset $\mathcal{O} \subset \mathcal{O}_{0,1}$ such that problem (5.1) has at least two positive solutions for $(\lambda, \mu) \in \mathcal{O}$, at least one positive solution for $(\lambda, \mu) \in\left(\mathcal{O}_{0,1} \backslash \mathcal{O}\right) \cup \Gamma_{0}$, and no solution for $(\lambda, \mu) \in \mathcal{O}_{0,2}$.

If $k_{i}:\left(l_{1}, l_{2}\right) \rightarrow(0, \infty)$ are continuous and singular at $r=l_{1}$ and/or $l_{2}$, then $h_{i}$ are also singular at $t=0$ and/or 1 . In this case, we assume

$$
\int_{l_{1}}^{l_{2}}\left(r^{(2-n)}-l_{1}^{(2-n)}\right)\left(l_{2}^{(2-n)}-r^{(2-n)}\right) k_{i}(r) d r<\infty
$$

then we can easily check that both $h_{i}$ satisfy $\left(D_{1}\right)$ and apply Theorem 4.1 to obtain the following corollary. 
Corollary 5.2. Assume $\left(D_{2}\right) \sim\left(D_{6}\right)$. If both $k_{i} \in C\left(\left(l_{1}, l_{2}\right),(0, \infty)\right)$ satisfy

$$
\int_{l_{1}}^{l_{2}}\left(r^{2-n}-l_{1}^{2-n}\right)\left(l_{2}^{2-n}-r^{2-n}\right) k_{i}(r) d r<\infty
$$

Then the conclusion of Corollary 5.1 is valid.

\subsection{On an Exterior Domain}

Let us consider

$$
\begin{gathered}
\Delta u+\lambda k_{1}(|x|) f(u, v)=0, \\
\Delta v+\mu k_{2}(|x|) g(u, v)=0, \quad|x|>r_{0}, \quad|x| \neq r_{1}, \\
\left.\Delta u\right|_{|x|=r_{1}}=I_{u}\left(\left.u\right|_{|x|=r_{1}}\right),\left.\quad \Delta v\right|_{|x|=r_{1}}=I_{v}\left(\left.v\right|_{|x|=r_{1}}\right), \\
\left.\frac{\Delta \partial u}{\partial r}\right|_{|x|=r_{1}}=\frac{n-2}{r_{0}}\left(\frac{r_{1}}{r_{0}}\right)^{1-n} N_{u}\left(\left.u\right|_{|x|=r_{1}}\right), \\
\left.\frac{\Delta \partial v}{\partial r}\right|_{|x|=r_{1}}=\frac{n-2}{r_{0}}\left(\frac{r_{1}}{r_{0}}\right)^{1-n} N_{v}\left(\left.v\right|_{|x|=r_{1}}\right), \\
u(x)=a \geq 0, \quad v(x)=b \geq 0, \quad \text { if }|x|=r_{0}, \\
u(x) \rightarrow c>a, \quad v(x) \rightarrow d>b, \quad \text { as }|x| \rightarrow \infty,
\end{gathered}
$$

where $f(0,0)=0, g(0,0)=0,0<r_{0}<r_{1}$, and $n>2$. Assume that both $k_{i}:\left[r_{0}, \infty\right) \rightarrow(0, \infty)$ are continuous. Applying changes of variables, $r=|x|$ and $t=1-\left(r / r_{0}\right)^{2-n}$, we may transform problem (5.4) into problem (3.20), where $t_{1}=1-\left(r_{0} / r_{1}\right)^{n-2}$ and $h_{i}$ are written as

$$
h_{i}(t)=\frac{r_{0}^{2(n-1)}}{(n-2)^{2}}(1-t)^{-2(n-1) /(n-2)} k_{i}\left(r_{0}(1-t)^{-1 /(n-2)}\right) \text {. }
$$

We know that $h_{i}$ are singular at $t=1$ and can easily check that $h_{i}$ satisfy $\left(D_{1}\right)$ if $k_{i}$ satisfy $\int_{r_{0}}^{\infty} r k_{i}(r) d r<\infty$ for $i=1,2$. Thus by Theorem 4.1, we obtain the following result.

Corollary 5.3. Assume $\left(D_{2}\right) \sim\left(D_{6}\right)$. If both $k_{i} \in C\left(\left[r_{0}, \infty\right),(0, \infty)\right)$ satisfy

$$
\int_{r_{0}}^{\infty} r k_{i}(r) d r<\infty
$$

then the conclusion of Corollary 5.1 is valid for problem (5.4). 


\section{Acknowledgment}

This work was supported for two years by Pusan National University Research Grant.

\section{References}

[1] S. C. Hu and V. Lakshmikantham, "Periodic boundary value problems for second order impulsive differential systems," Nonlinear Analysis: Theory, Methods E Applications, vol. 13, no. 1, pp. 75-85, 1989.

[2] S. G. Hristova and D. D. Baĭnov, "Existence of periodic solutions of nonlinear systems of differential equations with impulse effect," Journal of Mathematical Analysis and Applications, vol. 125, no. 1, pp. 192-202, 1987.

[3] E. K. Lee and Y.-H. Lee, "Multiple positive solutions of a singular Gelfand type problem for secondorder impulsive differential systems," Mathematical and Computer Modelling, vol. 40, no. 3-4, pp. 307328, 2004.

[4] B. Liu and J. Yu, "Existence of solution of $m$-point boundary value problems of second-order differential systems with impulses," Applied Mathematics and Computation, vol. 125, no. 2-3, pp. 155175, 2002.

[5] L. Liu, L. Hu, and Y. Wu, "Positive solutions of two-point boundary value problems for systems of nonlinear second-order singular and impulsive differential equations," Nonlinear Analysis: Theory, Methods \& Applications, vol. 69, no. 11, pp. 3774-3789, 2008.

[6] Y. Dong, "Periodic solutions for second order impulsive differential systems," Nonlinear Analysis: Theory, Methods \& Applications, vol. 27, no. 7, pp. 811-820, 1996.

[7] Y.-H. Lee, "A multiplicity result of positive radial solutions for a multiparameter elliptic system on an exterior domain," Nonlinear Analysis: Theory, Methods \& Applications, vol. 45, pp. 597-611, 2001.

[8] D. J. Guo and V. Lakshmikantham, Nonlinear Problems in Abstract Cones, vol. 5, Academic Press, Boston, Mass, USA, 1988.

[9] G.-M. Cho and Y.-H. Lee, "Existence and multiplicity results of positive solutions for Emden-Fowler type singular boundary value systems," Dynamics of Continuous, Discrete E Impulsive Systems, vol. 12, no. 1, pp. 103-114, 2005. 